\title{
Zur Frage der Zulassung von Küchenkehrichtzerkleinerungsapparaten (Küchenmühlen)
}

\author{
Vortrag, gehalten anlässlich eines Kolloquiums \\ der Hydrobiologischen Kommission der Schweiz. Naturforschenden Gesellschaft \\ am 20. Mail I95S \\ Von Dipl.-Ing. A. Hör LER \\ Chef der bautechnischen Abteilung der Eidg. Anstalt für Wasserversorgung, \\ Abwasserreinigung und Gewässerschutz an der ETH, Zürich \\ (Mitteilung Nr. 92 der EAWAG.) \\ Eingegangen am 30. Mai I955
}

\begin{abstract}
A. Einleitung
Von Amerika aus wurde in den letzten Jahren der europäische Markt in zunehmendem Masse mit Apparaten beliefert, die der Zerkleinerung der Küchenabfälle dienen, derart, dass diese direkt der Kanalisation übergeben und abgeschwemmt werden können. Mit den Vor- und Nachteilen dieser Küchenkehrichtzerkleinerungsapparate hatten sich zuerst die für den Gewässerschutz von Gesetzes wegen verantwortlichen Instanzen auseinanderzusetzen. Auf Grund einer solchen Beurteilung wurde die Installation der Apparate daraufhin erlaubt, geduldet oder verboten. Letzteres ist zum Beispiel im Kanton Zürich und in den Städten Basel, Bern und Luzern der Fall. Wie bei allen Neuerungen, treten bei der Beurteilung dieser Küchenkehrichtzerkleinerungsapparate gewisse Unsicherheiten oderWidersprüche in den Auffassungen auf, die eine Ưber- bzw. Unterbewertung verschiedener Faktoren und Gesichtspunkte zur Folge haben können. Einerseits werden diese Zerkleinerungsapparate als wichtigste Erfindung seit der Einführung des WC. und der Schwemnkanalisation gepriesen, anderseits als unerwünschte Komplikation der Abwassertechnik verdammt. Es ist deshalb zu verstehen, dass anlässlich der letztjährigen Delegiertenversammlung der Schweizerischen Vereinigung für Gewässerschutz (SVGS.)
\end{abstract}


der Wunsch ausgesprochen wurde, es möchte vom gesamtschweizerischen Standpunkt aus geprüft werden, ob und unter welchen Umständen die Küchenkehrichtzerkleinerungsapparate zugelassen werden könnten.

\section{B. Wie sehen diese Apparate aus und welche Eigenschaften haben sie?}

Die Küchenkehrichtzerkleinerungsapparate werden auch als Küchenkehrichtvertilger oder als Abfallvertilger schlechthin bezeichnet. Der Einfachheit halber seien diese im folgenden «Küchenmühlen» genannt. Sie dienen zur Zerkleinerung und Abschwemmung von Gemüseabfällen, Früchteschalen, Fischgräten, Knochen, Zigarren- und Zigarettenstummeln usw. Gegenstände aus Glas, Metall, Leder, Gummi, Stanniol und Textilien dürfen der Küchenmühle nicht zugeführt werden.

Die Küchenmühle wird unter dem Abwaschtrog oder Schüttstein ein-

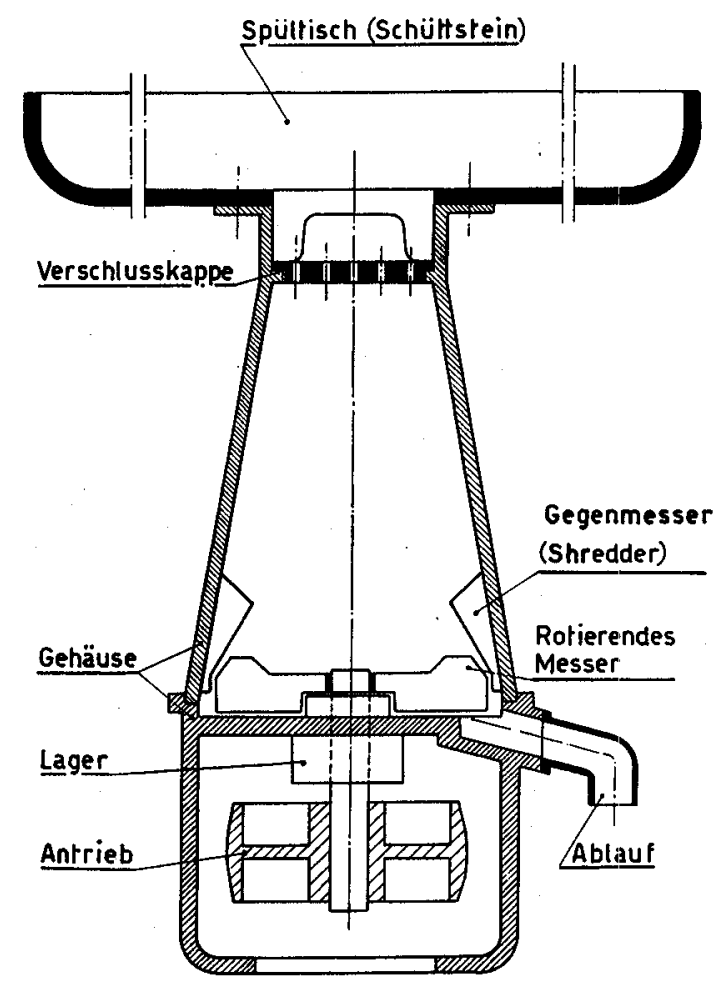

Abbildung 1 
gebaut. Das Gerät weist einen grössten Gehäusedurchmesser von rund $20 \mathrm{~cm}$ und eine Höhe von rund $35 \mathrm{~cm}$ auf. Es kann zum Beispiel ein auf Kugellagern laufendes Schwungrad mit Schneideelementen besitzen, das durch einen Motor von $1 / 4$ bis $1 / 2$ PS Leistung angetrieben wird. Eine bestimmte Fabrikmarke der Küchenmühle arbeitet nur, wenn während des Zerkleinerungsvorganges mindestens. $8 \mathrm{l} / \mathrm{min}$ Wasser zugegeben werden.

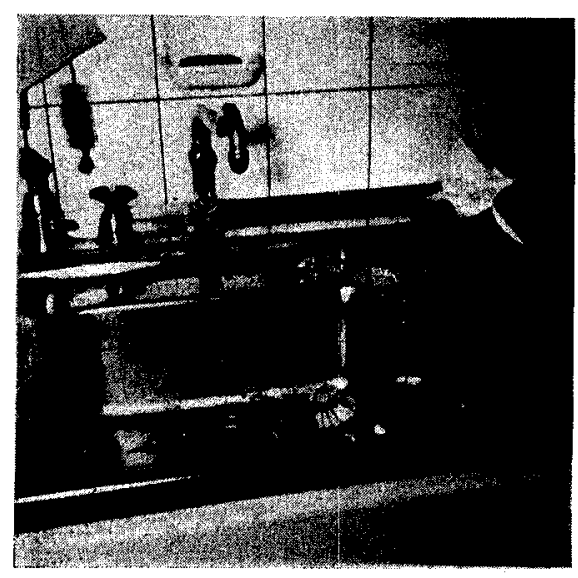

Abbildung 2

Wird diese Wasserzugabe gedrosselt oder ganz abgestellt, so stellt auch der Motor der Küchenmühle automatisch seine Tätigkeit ein. Andere Betriebsarten sind bei anderen Marken möglich.

Mit dem Anfall an Küchenkehricht eines normalen Haushaltes wird die Küchenmühle innert weniger Minuten fertig. Bei gefüllter Mühle geht der Zerkleinerungsvorgang rasch vor sich, während es einer gewissen Zeit bedarf, bis die letzten Reste zerkleinert und abgespült sind.

Ein Schnittdurch eineKüchenmühle ist aus Abbildung I ersichtlich. Dieses System besteht zum Beispiel aus einem Tragring (in dem die Verschlusskappe montiert wird); dem Hohlraum zur Aufnahme des Küchenkehrichtes zwischen der Verschlusskappe und dem Messer, dem rotierenden und mit Schneidemessern versehenen Impeller mit dem « Shredder» als Gegenmesser, dem vertikalachsigen Elektromotor und dem Ablaufstutzen mit Anschluss an die Kanalisation. Andere Apparate arbeiten mit Schlagwerkzeugen nach Art der Hammermühlen. 
Abbildung 2 zeigt, wie die Resten, die von der Zubereitung des Mahles übrigbleiben, in die Öffnung der Küchenmühle befördert werden. Der Verschluss der Öffnung ist weggenommen und befindet sich auf dem Rand des Abwaschtroges. Rechts oben ist der Schalter für die Inbetriebsetzung des Motors sichtbar.

Nach Einbringung des Kehrichtes in die Küchenmühle wird die Ver-

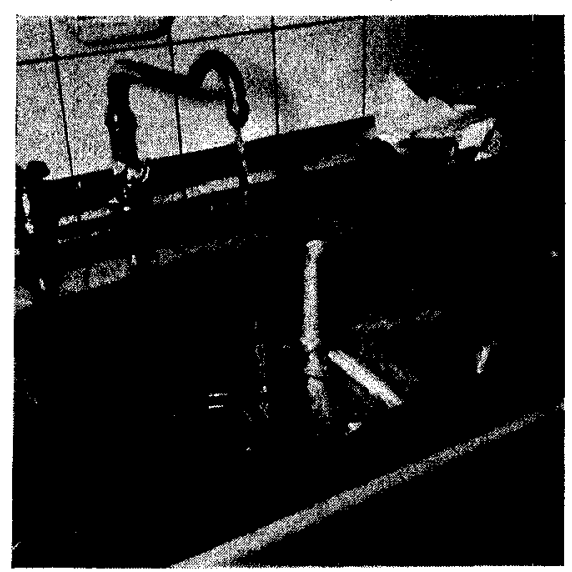

Abbildung 3

schlusskappe eingesetzt und bei dieser amerikanischen Ausführung auf «ON» gebracht (Abb. 3). Beim Aufdrehen des Wasserhahns nimmt der Motor den Mahlvorgang auf, sobald die angegebene minimale Wassermenge zugegeben wird. Nach Beendigung des Mahlvorganges, der bei einiger Übung am verminderten Geräusch des Apparates erkannt wird, ist Motor und Wasserhahn abzustellen.

Der Einbau des Apparates unter dem Abwaschtrog ist aus Abbildung 4 ersichtlich. Tablare können trotzdem unter dem Schüttstein angebracht werden, sofern der Platz für die Küchenmühle ausgespart wird.

Die Anschaffungskosten eines Apparates belaufen sich auf Fr. 450.- für das billigste Aggregat. Fertig montiert und angeschlossen ist unter günstigen Verhältnissen mit Fr. 600.- bis Fr. 700.- zu rechnen. Die Betriebskosten ohne Amortisation und Reparaturen ermitteln sich wie folgt:

a) Wasserverbrauch für eine vierköpfige Familie rund 8-ro 1/min wäh- 
rend 4-5 min pro Tag bzw. rund Iol pro Bewohner und Tag oder rund $3,6 \mathrm{~m}^{3}$ pro Bewohner und Jahr à 20 Rappen $=\quad \mathrm{ca}$. Fr. $-.80 / \mathrm{Jahr}$

b) Elektrische Energie bei einem Einheitspreis

von 7 Rappen/kWh, aufgerundet ca.

Total pro Bewohner und Jahr somit

,$-.70 / \mathrm{Jahr}$

ca. Fr. I.50.

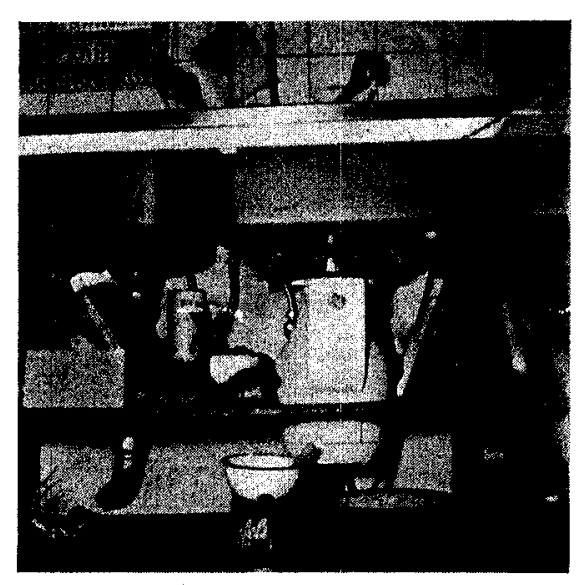

Abbildung 4

\section{Welche Auswirkungen hat die Einführung der Küchenmühlen auf die Abwasseranlagen und die Vorfluter?}

Die Vor- und Nachteile der Einführung der Küchenmühlen können unter den verschiedensten Aspekten betrachtet werden, zum Beispiel unter hygienischen Gesichtspunkten, von wirtschaftlichen Erwägungen aus, wie zum Beispiel die Verminderung der Kehrichtabfuhr und anderes mehr.

Im folgenden wird der Schwerpunkt auf die Betrachtung der Auswirkungen auf die Abwasseranlagen bzw. auf den Gewässerschutz gelegt, während die übrigen Gesichtspunkte nur gestreift werden sollen.

\section{Auswirkungen auf die Kanalisationen}

Der Einfluss der Küchenmühlen auf die Kanalisationen wird in der Fachwelt sehr verschieden beurteilt. Äusserste Bedenken wegen Verstopfungsgefahr der Kanalisationen durch den Küchenkehricht auf der einen Seite 
bis zur Verneinung aller Schwierigkeiten auf der andern Seite sind der Fachliteratur zu entnehmen.

Es scheint mir richtig zu sein, dass in einem nach neuzeitlichen Gesichtspunkten ausgebauten und durchkonstruierten Kanalisationsnetz durch die Zunahme der Abwasser- und Stoffmenge keine Betriebserschwernisse zu gewärtigen sind und dass die Kanalquerschnitte nicht vergrössert werden müssen. Einzig in den Endsträngen der Kanalisation ist eine vermehrte Depotbildung durch das zusätzliche Küchengut möglich. In alten Kanalnetzen, bei Schächten mit Säcken, namentlich auch bei ungenügendem Gefälle oder unter Umständen sogar bei Gegengefälle werden allerdings vermehrte Ablagerungen bei Einführung der Küchenmühlen zu erwarten sein, die eine zusätzliche Reinigungsarbeit seitens des Kanaldienstes erfordern.

Durch die wesentlichen Sandmengen, die zusammen mit dem Küchenkehricht in die Kanalisationen gelangen, ist auch eine vermehrte mechanische Abnützung der Kanalsohlen möglich.

\section{Auswirkungen auf die Kläranlagen}

Die Literaturangaben $[\mathrm{r}, 2,3,4,5,6]^{1}$ ) über die Veränderung der Eigenschaften des Abwassers nach Einführung der Küchenmühlen, die massgebend für die Beurteilung des Einflusses dieser Apparate auf die Kläranlagen sind, weichen stark voneinander ab. Folgende Daten, die zum Teil von der Abteilung Wasserbau und Wasserrecht der Kantonalen Baudirektion Zürich zusammengestellt wurden, dürften den Verhältnissen entsprechen, wie sie in den Vereinigten Staaten anzutreffen sind:

a) Zunahme des täglichen Abwasseranfalles rund 8-ro 1/Einwohner.

b) Täglicher Anfall an Küchenkehricht $100-400 \mathrm{~g} /$ Einwohner (extrem $900 \mathrm{~g}$ ), mit einem Wassergehalt von $75-85 \%$. Im Winter gilt die kleinere, im Sommer die grössere Gewichtszahl.

c) Trockengewicht des Kehrichts 20-130 g/Einwohner und Tag, im Mittel etwa $55 \mathrm{~g} /$ Einwohner und Tag.

d) Zunahme des Sandgehaltes des Abwassers um 20-40\%.

e) Die geringe Zunahme des Rechengutes dürfte zu vernachlässigen sein.

f) Der Gehalt an absetzbaren Stoffen nimmt um $15-50 \%$ zu (extrem $100 \%$ ).

1) Die eingeklammerten Zahlen beziehen sich auf das Literaturverzeichnis am Schluss der Abhandlung. 
g) Zunahme der Schlammenge um I5-30\%.

h) Verbesserung des Kläreffektes der Absetzbecken durch die Kehrichtzugabe um $2-5 \%$.

i) Der biochemische Sauerstoff bedarf (BSB5) des Rohwassers weist eine Zunahme um $30-50 \%$ auf (extrem 100\%).

k) Die Zunahme der Konzentration des in Absetzbecken geklärten Abwassers beträgt rund $5-20 \%$.

1) Erhöhter Gasanfall 100\%, extrem $250 \%$.

Die oben bezeichneten Zunahmen wären dann zu erwarten, wenn die Küchenmühlen in sämtlichen Haushaltungen installiert würden. Nach einer Veröffentlichung von HASELTINE [I] ist jedoch mit einem Sättigungsgrad zu rechnen, der dann erreicht sein dürfte, wenn etwa $60 \%$ sämtlicher Haushaltungen Küchenmühlen besitzen. Für unsere Verhältnisse dürfte der angegebene Sättigungswert wesentlich niedriger sein, sofern nicht, wie im Fall der Stadt Jaspar (Indiana), die Einführung der Küchenmühlen als obligatorisch erklärt wird.

Der geringe zusätzliche Wasserbedarf bei Einführung der Küchenmühlen würde an und für sich kaum eine Vergrösserung der Kläranlage nahelegen. Der vermehrte Sandanfall bedingt auch keine Vergrösserung des Sandfanges, hingegen wird häufigere Entleerung nicht zu umgehen sein. Bei Neukonstruktionen wird man den Sandstapelraum entsprechend grösser vorsehen. Beim Rechen wird etwas Mehrarbeit für die Beseitigung des Gutes zu gewärtigen sein, sofern keine Komminutoren oder ähnliche Apparate eingebaut sind. Vorhandene getrennte Absetzbecken mit knapp dimensionierten Schlammsammelräumen müssen öfteren Schlammablass erhalten, ohne dass das Absetzbecken selbst vergrössert werden müsste. Die Zunahme des Schlammvolumens und namentlich die Zunahme der Abwasserkonzentration haben eine wesentliche Vergrösserung der Faulräume samt Schlammbehandlung sowie des biologischen Teils der Abwasserreinigungsanlage, mit Ausnahme der Nacbklärbecken, zur Folge. Bei bestehenden Abwasserreinigungsanlagen äussert sich die Einführung der Küchenmühlen in dem Sinne, dass frühzeitiger als beabsichtigt an die Erweiterung der Reinigungsanlage gedacht werden muss und dass für die zweite Ausbauetappe mit grösseren Volumen der erwähnten Bauwerke zu rechnen sein wird. Die Angaben über die erforderliche Vergrösserung dieser Anlageteile infolge Installation von Küchenmühlen schwanken von $S$ bis $50 \%$. Dabei ist aus den Literaturangaben nur selten ersichtlich, mit welchen Sättigungsziffern für die Einführung der Apparate gerechnet 
wurde. Es ist ebenfalls zu berücksichtigen, dass namentlich die Betriebskosten für den biologischen Teil (Mehrkosten für Druckluft bei Verwendung des Belebtschlammverfahrens) entsprechend anwachsen sowie die Kosten für die Schlammbehandlung. Als zusätzliche Einnahmen kann der vermehrte Gasanfall in Rechnung gesetzt werden, der nach ToLMAN [2] bis zu 100\% und mehr beträgt. Durch die Zugabe des Kehrichtes in die Faulräume soll allerdings die Gasqualität leiden, was jedoch von den Gaswerken begrüsst werden dürfte. Zudem wurden Betriebserschwernisse durch stärkere Schwimmdeckenbildungen in den Faulräumen festgestellt.

\section{Auspirkung auf die Vorfluter}

So mannigfaltig die Anordnung der Kanalisationsnetze ist, so mannigfaltig ist auch der Einfluss der Installation der Küchenmühlen und des dadurch in die Kanäle abgeschwemmten Kehrichtes auf die Vorfluter.

Betrachten wir vorerst den Fall der direkten Entwässerung in den Vorfluter ohne Zwischenschaltung einer Kläranlage, wieer in Abbildung 5 dargestellt ist. Dieser Fall, der leider noch häufig angetroffen wird, ist selbst bei grossen und leistungsfähigen Vorflutern als unzulässig zu bezeichnen. Es ist klar, dass im Falle der Abschwemmung sämtlicher Abfallstoffe in den Vorfluter jede zusätzliche Belastung desselben vermieden werden muss.

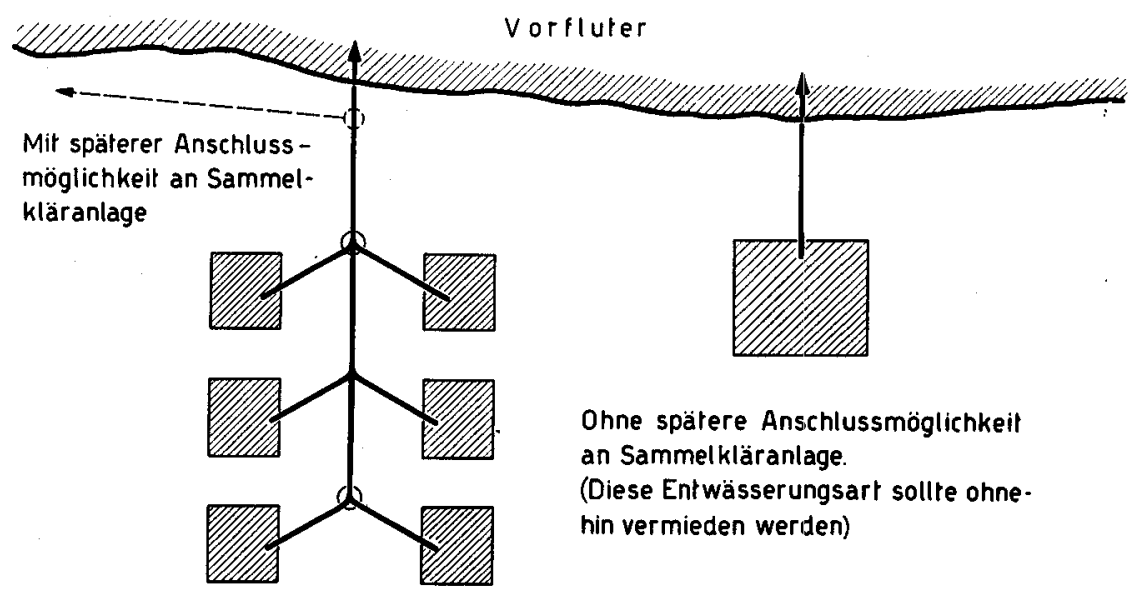

Installation von Küchenmühlen nicht zulässig, solange nicht eine geregelte Ent wässerung mil Kläreinrichtungen erstellt wird. 
Eine Installation von Küchenmühlen könnte in solchen Fällen meines Erachtens nicht verantwortet werden.

Sofern die Liegenschaften Hauskläranlagen besitzen, wie dies in Abbildung6dargestellt ist, dürften in der Mehrzahl derFälle dieKüchenmühlen als zulässig bezeichnet werden können. Es ist jedoch zu beachten, dass die Schlammenge vermehrt wird und dadurch bei Neuanlagen die Faulräume vergrössert werden müssen. Bei bestehenden Kläreinrichtungen wird eine

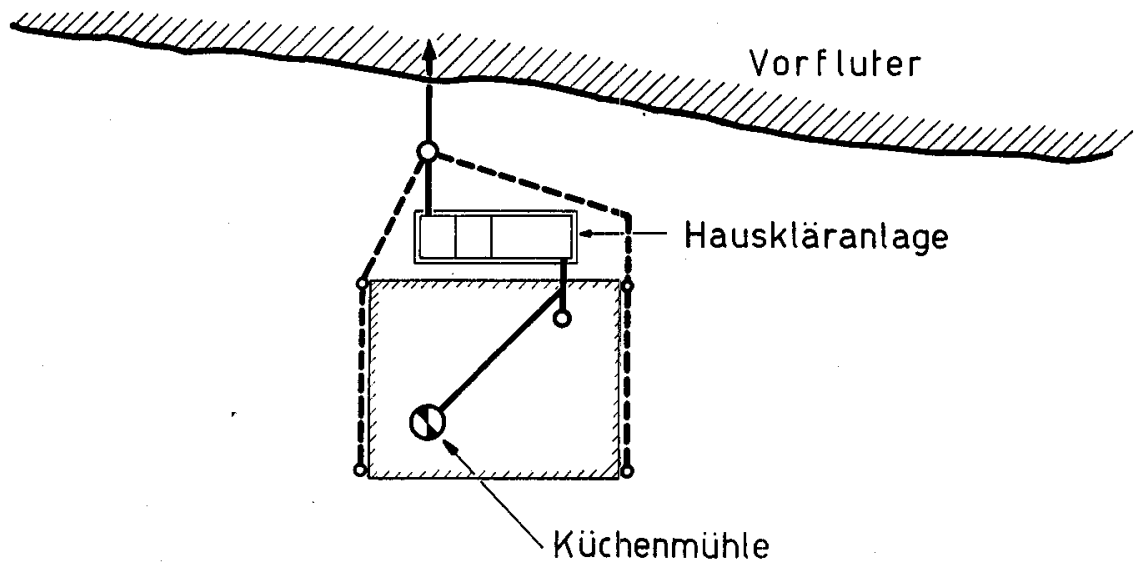

Installation von Küchenmühlen zulässig bei grösseren Abmessungen der Hauskläranlage oder bei späłerer Anschlussmöglichkeil an Sammelkläranlage und häufiger Entleerung der Einzelanlage.

Abbildung 6

häufigere Entleerung ins Auge zu fassen sein. Nachteilig ist der grössere Sandanfall, ebenso die grössere Abwasserkonzentration, die jedoch bei den kurzen Transportdistanzen zwischen Küche und Kläranlage kaum die hohen Werte erreichen dürfte wie im Falle zentraler Reinigungsanlagen.

Sind die Küchenabwässer an Fettabscheider angeschlossen, so ist von der Installation der Küchenmühlen abzusehen. In Restaurants und Hotels kommen die Küchenmühlen wegen der Intensität des Küchenbetriebes ohnehin nicht in Frage, sofern nicht grössere Modelle auf den Markt gelangen.

Die Entwässerungsverfahren lassen sich, wie bekannt, in zwei grund- 
sätzlich verschiedene Systeme einteilen: in das Trenn- und das Mischsystem. Beim Trennsystem werden Schmutz- und Regenwasser in zwei voneinander vollständig getrennten Leitungsnetzen gesammelt und abgeleitet. Bei Einführung der Küchenmühlen in Gemeinden, die nach dem Trennsystem entwässert werden (Abb. 7), wird deshalb das Küchenabwasser vor Erreichung der Kläranlage mit dem Vorfluter unter keinen Umständen in Berührung kommen. Stellt man an die Sammelreinigungs-

(1) Küchenmühlen

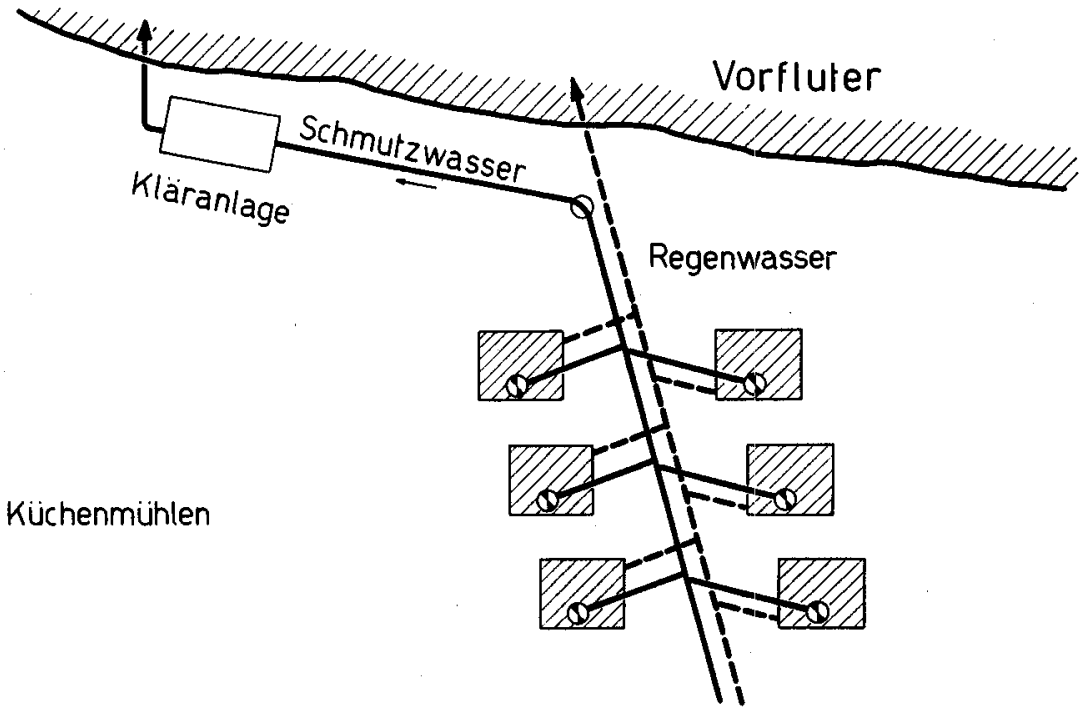

Insiallation von Küchenmühlen zulässig, bei entsprechender Vergrösserung

der Kläraniage

Abbildung 7

anlage die Forderung, dass die Bauwerke entsprechend ihrer zu erwartenden zusätzlichen Belastung dimensioniert werden, so dass die Abflusskonzentration trotz des Einbaues der Küchenmühlen nicht grösser wird als vorher, so dürfte gegen die Einführung dieser Apparate nichts einzuwenden sein.

Beim Miscbsystem (Abbildung 8), das in der Schweiz das bevorzugte Entwässerungssystem ist, werden die Regenmassen zusammen mit dem wesentlich geringeren, aber stetig fliessenden Schmutzwasseranteil im selben Rohrstrang abgeleitet. Aus wirtschaftlichen Gründen werden an bestimmten, zweckmässig gewählten Stellen zur Entlastung des Kanalnetzes soge- 
nannte Regenauslässe erstellt. Bei Starkregen werden die anfallenden Regenwasserspitzen, die selbstverständlich mit Schmutzwasser vermengt sind, direkt dem Vorfluter übergeben. Zur Verbesserung der Vorfluterverhältnisse, namentlich in ästhetischer Hinsicht, werden bei Regenauslässen in bestimmten Fällen Rechen, Siebe oder Regenwasserklärbecken angeordnet. Durch die Zunahme der absetzbaren und der gelösten Stoffe bei Einführung der Küchenmühlen dürfte die Vorfluterbelastung rund

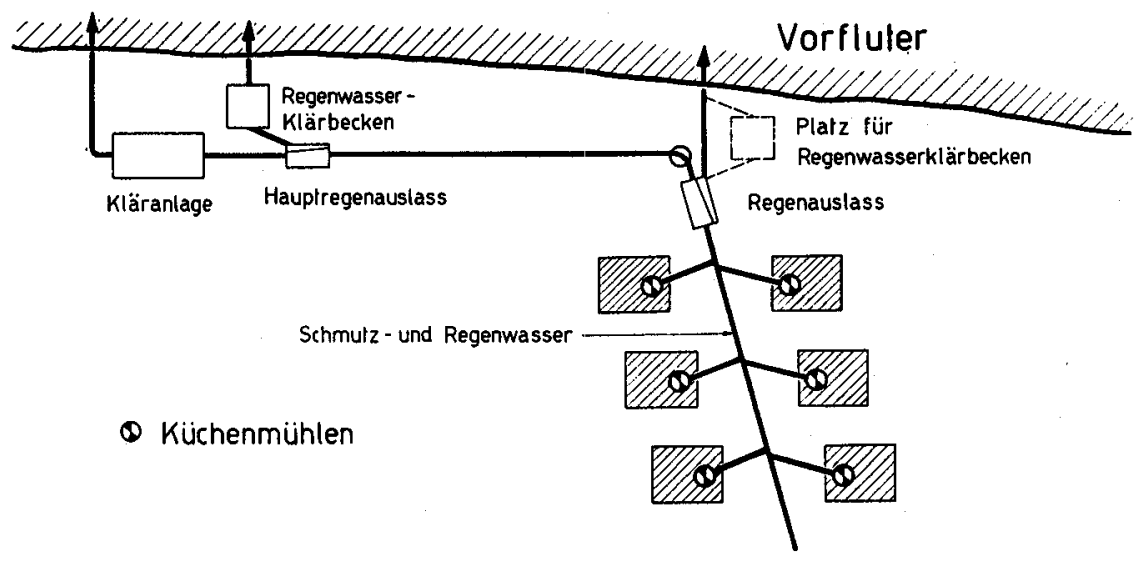

Installation von Küchenmühlen zulässig,sofern kritische Regenintensitäl beim Regenauslass erhöht werden kann und sofern Regenwasserklärbecken vor der Kläranlage und eventuell beim Regenauslass fruihzeiliger erstellt werden.

Abbildung 8

$50 \%$ grösser werden, falls in allen Haushaltungen diese Zerkleinerungsapparate installiert werden bzw. rund $30 \%$ bei einem Sättigungsgrad von $60 \%$. Die schärfste Forderung, die meines Erachtens gestellt werden kann, wäre die, dass nach Einführung der Küchenmühlen die Belastung der Vorfluter durch die Regenauslässe nicht grösser sein darf als vor Einführung der Apparate. Diese Forderung ist durch zusätzliche Erstellung von Regenwasserklärbecken üblicher Grösse und Bauart nicht zu erreichen. Hingegen könnte, und wie mir scheint wirtschaftlicher, diese Forderung durch Erhöhung der kritischen Regenintensität, bei der ein Regenauslass zu spielen beginnt, eingehalten werden. Dadurch wird jedoch der Dimensionierung und Ausbildung des Hauptregenauslasses vor der Kläranlage vermehrte Beachtung geschenkt werden müssen. 
Da die erwähnte Forderung weitreichende finanzielle Konsequenzen haben kann, wurde an Hand eines praktischen Beispiels versucht, die erforderliche Erhöhung der kritischen Regenintensität rechnerisch festzulegen, um feststellen zu können, ob für den Schutz des Vorfluters eine wirtschaftlich tragbare Erhöhung der kritischen Abwassermenge genügen wird oder nicht.

Für die Beurteilung des Einflusses von Regenauslässen auf Vorfluter

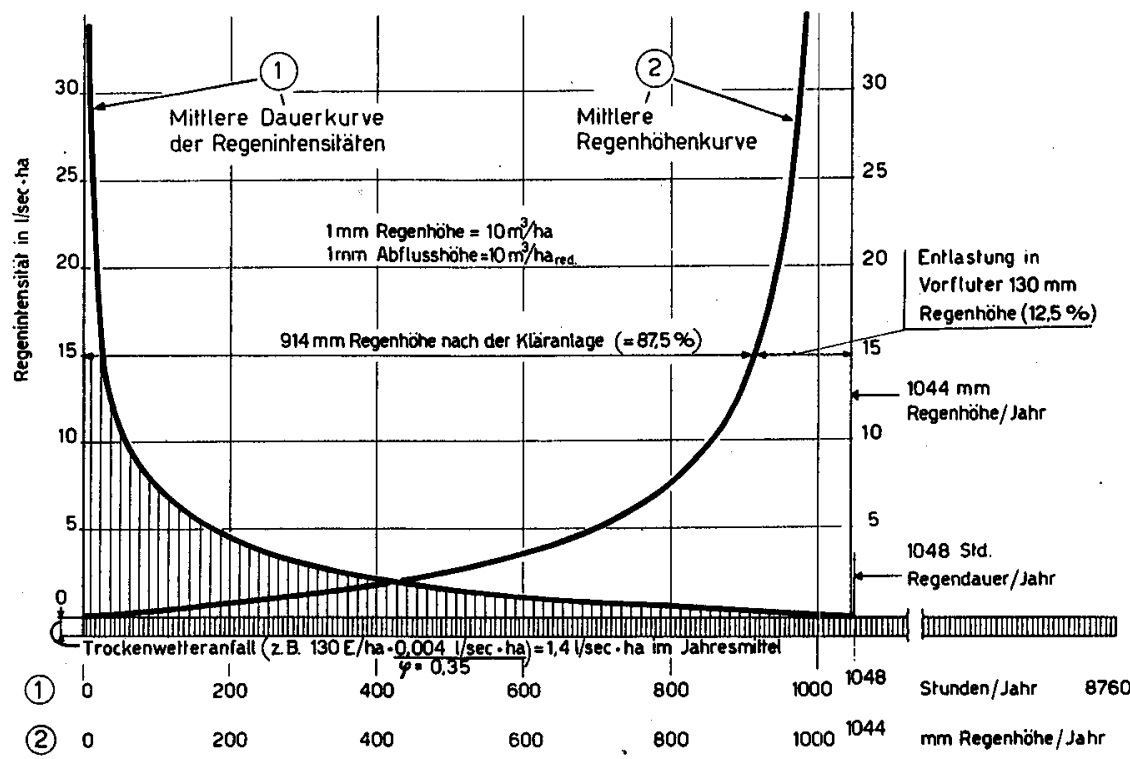

Abbildung 9

dienen die Dauerkurven der Regenintensitäten und die Regenhöhenkurven, wie in Abbildung 9 dargestellt. Die Dauerkurve der Regenintensitäten gibt an, an wie vielen Stunden im Jahr Regenfälle bestimmte Intensitäten erreichen oder überschreiten. Aus der Regenhöhenkurve ist ersichtlich, welcher Anteil der jährlichen Regenhöhe bei Wahl einer bestimmten Regenintensität, der sogenannten kritischen, nach der Kläranlage weiterfliesst bzw. über den Regenüberfall in den Vorfluter gelangt. Bei Wahl einer kritischen Intensität von zum Beispiel I $5 \mathrm{l} / \mathrm{s}$ pro Hektare wird der dem schraffierten Teil der Dauerkurve der Regenintensitäten entsprechende Anteil (nebst dem als rechteckiges Band von der Abszisse nach unten aufgetragenen Schmutzwasseranteil) nach der Kläranlage geleitet. Zur Entlastung in den Vorfluter gelangen somit nur $12,5 \%$ des Regenabflusses, wie aus 
der Darstellung der Regenhöhenkurve hervorgeht. Das Beispiel beruht auf der Annahme eines Baugebietes mit einer Besiedlungsdichte von I30 Einwohner pro Hektare und einem Abflussbeiwert von 0,35 , entsprechend der Wohnzone W 2 I/3 der Stadt Zürich, mit zwei Vollgeschossen und einem ausgebauten Dachstock.

Um zu überprüfen, ob die angegebene Form der Dauerkurve der Regenintensität nur für Zürich und Umgebung gilt oder ob die Form als für

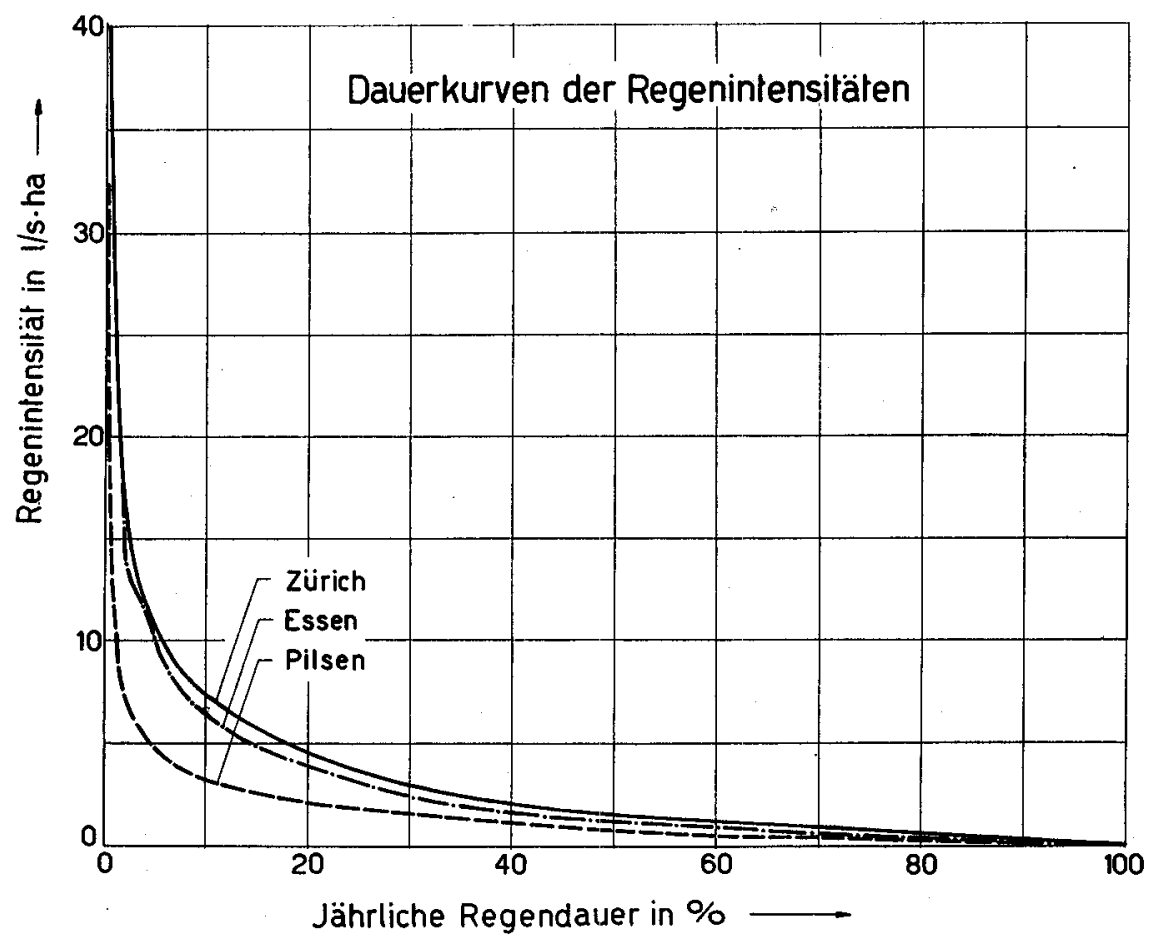

Abbildung 10

ein grösseres Gebiet charakteristisch bezeichnet werden darf, sind in Abbildung Io die Dauerkurven der Regenintensitäten für Essen [7], Pilsen [8] und Zürich [9] dargestellt, wobei die jährliche Regendauer (Abszisse) je zu I00\% angenommen wurde. Aus der Darstellung ist ersichtlich, dass die Kurven - namentlich Essen und Zürich - relativ gut übereinstimmen, besonders wenn man bedenkt, dass keine Richtlinien über die einheitliche Auswertung der Regenstreifen bezüglich der Aufstellung von Dauerkurven bestehen. 
Nehmen wir nun die durch die Einführung von Küchenmühlen verursachte Erhöhung der Abwasserkonzentration entsprechend dem Gesagten zu Io bzw. 20 bzw. maximal $30 \%$ an, so können statt der Regenhöhenkurven auch Belastungskurven für den Vorfluter, gemessen am 5tägigen biochemischen Sauerstoff bedarf (BSBs), aufgetragen werden, die durch die Regenauslässe bei Wahl verschiedener Entlastungsintensitäten bedingt sind. Dabei wurde entsprechend den Beobachtungen von BRENTANo [Io] und MülLER-NeuHaUs [II] angenommen, dass die Mischwasserkonzentration bei der Entlastung von gleicher Grössenordnung sei wie die Konzentration des Trockenwetteranfalls. Diese Feststellung dürfte für grosse Kanalnetze mit geringem Gefälle gelten.

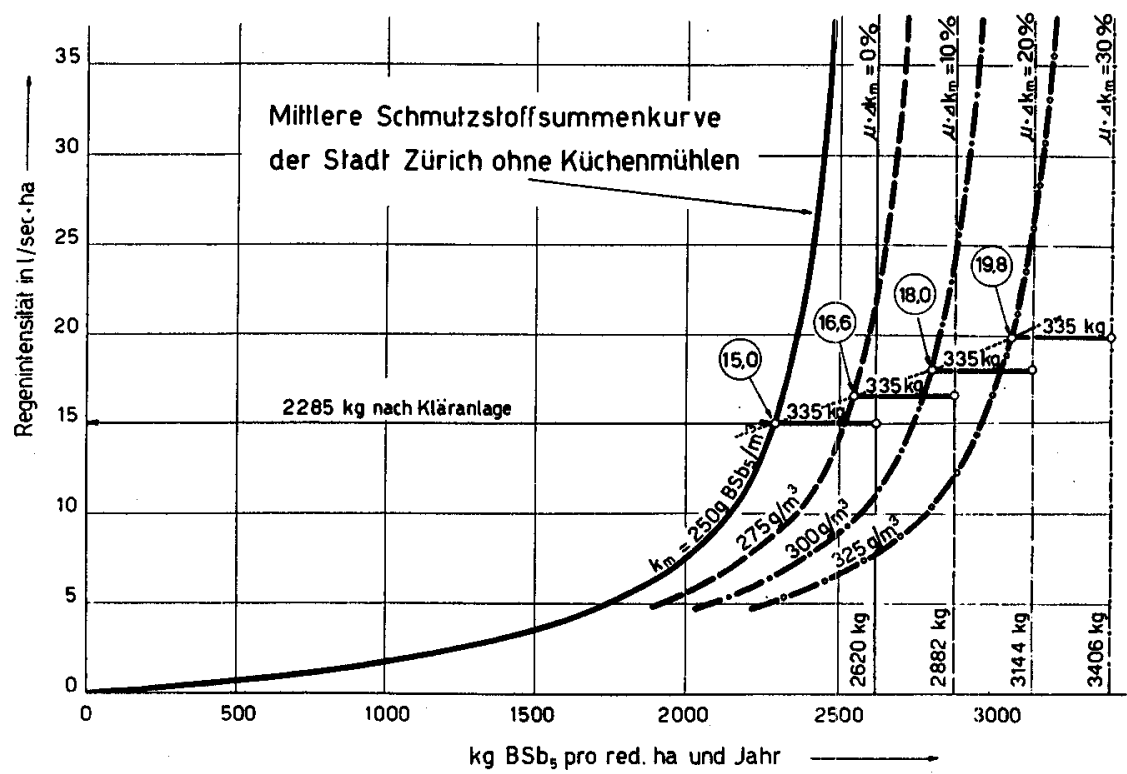

Abbildung 11

Unter den gemachten Annahmen gelangt von jeder reduzierten Hektare des Einzugsgebietes ohne installierte Küchenmühlen eine Schmutzstoffmenge entsprechend einem BSB5 von $335 \mathrm{~kg}$ pro Jahr bei Wahl einer kritischen Regenintensität von IS $1 / \mathrm{s}$ pro Hektare in den Vorfluter. In Abbildung 11 sind die Schmutzstoffsummenkurven für o, 10, 20 und 30\% Konzentrationszunahme des Abwassers aufgetragen und für jede Kurve jene Regenintensität ermittelt, bei der die jährliche Vorfluterbelastung $335 \mathrm{~kg}$ BSB5 pro angeschlossene reduzierte Hektare beträgt. 
Tabelle 1

\begin{tabular}{|c|c|c|}
\hline \multirow{2}{*}{$\begin{array}{c}\text { Zunahme } \\
\text { der Abwasserkonzentration } \\
\text { bei Einführungder Küchen- } \\
\text { mühlen um }\end{array}$} & \multicolumn{2}{|c|}{$\begin{array}{c}\text { Angenommene kritische Regenintensität bei 0 Prozzat } \\
\text { Konzentrationserhöhung }\end{array}$} \\
\cline { 2 - 3 } & $101 / \mathrm{s} / \mathrm{ha}$ & $15 \mathrm{sh} / \mathrm{a}$ \\
\hline $0 \%$ & $10,01 / \mathrm{s} / \mathrm{ha}$ & $15,0 \mathrm{l} / \mathrm{s} / \mathrm{ha}$ \\
$10 \%$ & $11,21 / \mathrm{s} / \mathrm{ha}$ & $16,61 / \mathrm{s} / \mathrm{ha}$ \\
$20 \%$ & $12,4 \mathrm{~s} / \mathrm{ha}$ & $18,2 \mathrm{l} / \mathrm{s} / \mathrm{ha}$ \\
$30 \%$ & $13,61 / \mathrm{s} / \mathrm{ha}$ & $19,81 / \mathrm{s} / \mathrm{ha}$ \\
\hline
\end{tabular}

Erforderliche Erhöhung der kritischen Regenintensität bei Zunahme der Abwasserkonzentration unter der Bedingung konstanter Vorfluterbelastung durch die Regenauslässe.

Die Ergebnisse dieser Ermittlung sind ausgeglichen in Tabelle I zusammengestellt, wobei die Zunahme der kritischen Regenintensitäten für die beiden Basiswerte von ro bzw. Is $1 / \mathrm{s}$ pro Hektare angegeben sind. Angenähert dürfte gelten, dass pro Prozent Konzentrationserhöhung des Abwassers die kritische Regenintensität, bei der ein Regenauslass entlasten darf, ebenfalls um rund $\mathrm{I} \%$ erhöht werden muss, damit die Schmutzstoffbelastung des Vorfluters konstant bleibt.

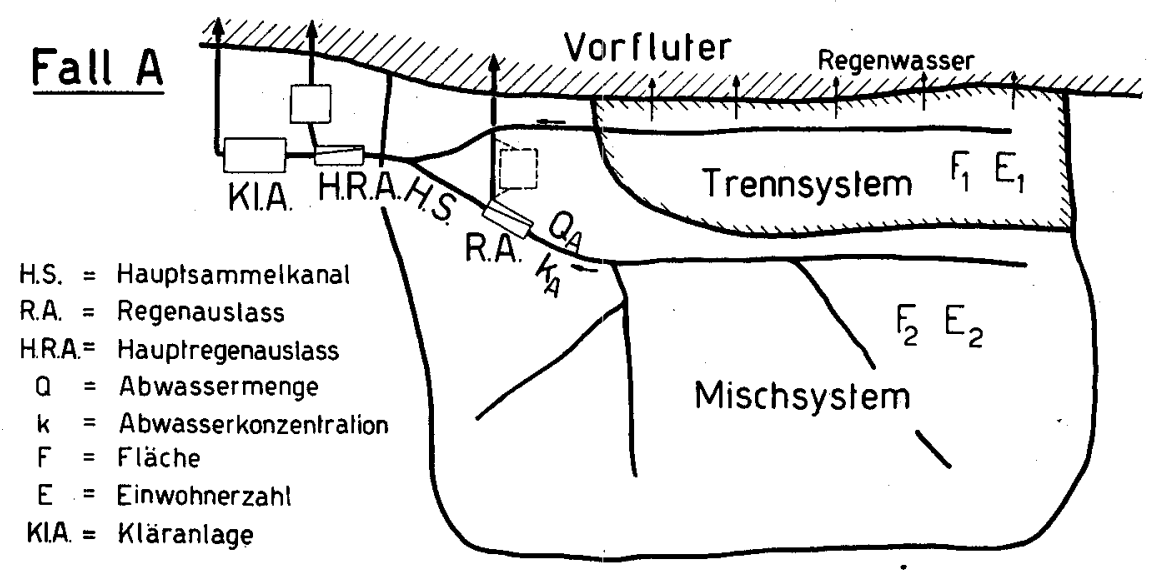

Installation von Küchenmühlen nach den gleichen Grundsätzen wie bei der Entwässerung im Mischsystem zulässig. 


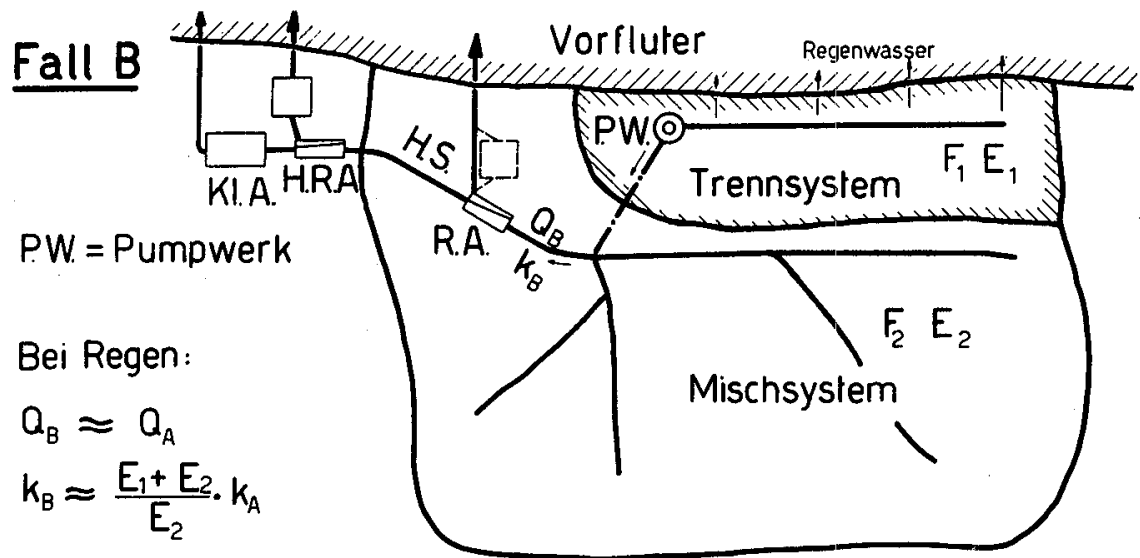

Installation von Küchenmühlen nach gleichen Grundsälzen wie bei Entwässerung im Mischsystem zulässig. Vermehrle Sandführung im Trennsystem bei Projektierung des Pumpwerkes berijcksichtigen,ebenso grössere Abwasserkonzentration gegenuiber Fall $A$ bei der Dimension. ierung des Regenauslasses!

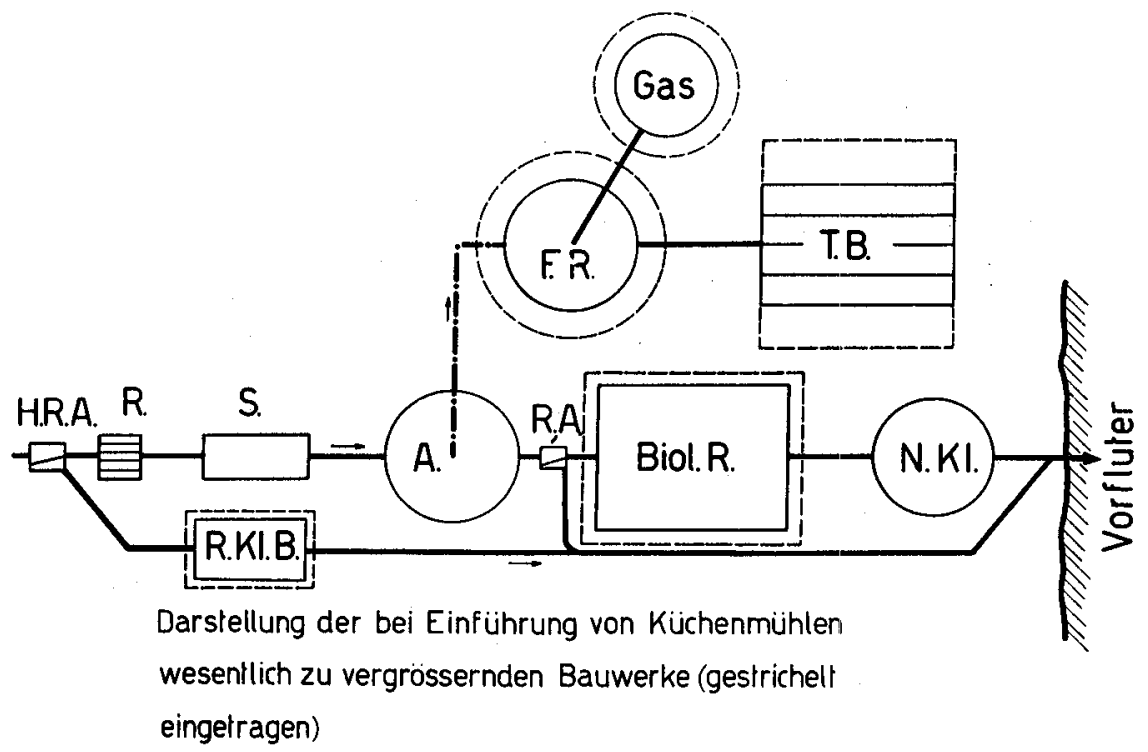


Die finanziellen Auswirkungen der Erhöhung der kritischen Regenintensität in angegebenen Ausmass dürfte tragbar sein, da sich die Kostenerhöhung praktisch nur für die an den Regenauslass direkt anschliessende nächst untere Teilstrecke auswirken wird.

Geben wir uns kurz Rechenschaft über die Auswirkungen der Küchenmühlen bei kombinierten Entwässerungssystemen, so dürfte unter anderen folgende technisch mögliche Lösung zur Beurteilung vorliegen (Abb.12): Teilgebiet mit Entwässerung im Trennsystem mit Anschluss unterhalb des letzten Regenauslasses an den Hauptsammelkanal. Hierbei gelten für die Trenn- bzw. Mischsystemgebiete die gleichen Richtlinien wie für die einzelnen Systeme selbst.

Abbildung 13 stellt den Fall eines Teilgebietes mit Entwässerung im Trennsystem dar, mit Anschluss oberhalb des letzten Regenauslasses an den Hauptsammelkanal, wobei die Möglichkeit maschineller Förderung des Schmutzwassers aus dem Trenn- in das Mischsystemgebiet angedeutet ist. Der vermehrten Sandführung wegen beim Einbau von Küchenmühlen ist dem Pumpwerk ein Sandfang vorzuschalten. Bei dieser Anordnung nimmt die Konzentration des Mischwassers (Schmutz- und Regenwasser) gegenüber dem vorhergehenden Bild (Fall A) zu, was bei der Erhöhung der kritischen Intensität von Einfluss ist.

Über die in einer Abwasserreinigungsanlage mit Einführung der Küchenmühlen zu vergrössernden Bauwerke gibt Abbildung 14 Auskunft. Die Bauwerksteile, die gestrichelt umrandet sind, stellen die wesentlich zu vergrössernden Bauteile dar.

\section{Zusammenfassung und Erwägungen: Welche Gesichtspunkte sind für die Zulassung der Küchenmühlen zu beachten?}

I. Die Beseitigung der Abfallstoffe ist ein vielschichtiges Gebiet von grösster hygienischer Bedeutung. Die Beseitigung der mit dem Abwasser anfallenden Schmutzstoffe im speziellen erfolgt heute durch die Erstellung von Entwässerungsnetzen mit Kläranlagen bzw. Abwasserreinigungsanlagen.

Eine geordnete Kehrichtbeseitigung erfolgt durch eine organisierte Kehrichtabfuhr. Anschliessend daran werden diese Abfallstoffe in mehr oder weniger einwandfreier Weise deponiert, verrottet oder verbrannt. Die Idee, den Kehricht mit dem Abwasserschlamm gemeinsam zu behandeln, ist nicht neu, wenn sie auch in der Schweiz noch ungebräuchlich ist. 
Für die Kehricht-Schlamm-Kombination bestehen zwei grundsätzlich verschiedene Möglichkeiten, nämlich:

a) Zugabe des Abwasserschlamms zum Kehricht, wonach eine gemeinsame Verrottung oder Verbrennung erfolgen kann.

b) Zugabe des Kehrichts zum Abwasser oder Abwasserschlamm.

Diese Zugabe zum Abwasser kann schon in der Küche durch Einbau der beschriebenen Küchenmühlen erfolgen oder durch grössere Zerkleinerungsmaschinen, die im Keller von Mehrfamilienhäusern bzw. an einzelnen zentral gelegenen Stellen in der Nähe der Hauptsammelkanäle oder auf der Kläranlage selbst installiert sind. Zudem wird die Auswaschung und Zerkleinerung des Kehrichts mit direkter Zugabe in den Faulraum der Kläranlagen ausgeübt.

Aus diesen Ausführungen ist ersichtlich, dass der Einbau von Küchenmühlen nur eine der verschiedenen Möglichkeiten zur gemeinsamen Beseitigung des Kehrichtes mit dem Abwasser darstellt. Da der Küchenkehricht nur ein Bruchteil des gesamten Kehrichts ausmacht, wird durch die Einführung von Küchenmühlen die Kehrichtabfuhr nicht überflüssig. Die Abfuhrkosten sollen sich sogar nur um etwa 10-20\% senken.

Wenn auch durch den Einbau der Küchenmühlen keine wesentlichen Einsparungen auf dem Sektor der Kehrichtbeseitigung erwartet werden kann, so stellen diese trotzdem eine zweckmässige und hygienisch einwandfreie Form der Beseitigung des Küchenkehrichts dar, so dass die Installation dieser Mühlen an und für sich begrüsst werden könnte, sofern die Abwasseranlagen gewisse, nachfolgend erwähnte Anforderungen erfüllen.

2. Die Voraussetzungen, unter denen die Küchenmühlen zugelassen werden könnten, sind etwa die folgenden, wobei kein Anspruch auf Vollständigkeit dieser Voraussetzungen erhoben wird:

a) Bei Liegenschaften mit Einzelentwässerung sollten genügend bemessene und einwandfrei gewartete Hauskläranlagen vorhanden sein.

b) Die Liegenschaften mit Anschluss an eine Sammelreinigungsanlage müssen durch ein einwandfrei ausgebautes Kanalnetz an eine zentrale Abwasserreinigungsanlage genügender Grösse angeschlossen sein. Der Ausbildung neuer bzw. der Verbesserung bestehender Regenauslässe ist besondere Beachtung zu schenken.

3. Nicht zuzulassen, wenigstens bis zur Behebung der Mißstände, wären die Küchenmühlen dort, wo schlecht angelegte Kanalisationsstränge, unterdimensionierte Regenauslässe oder überlastete Kläranlagen vor- 
handen sind. Unter Umständen kann sich das Verbot nur auf Teilgebiete mit schlechten Abflussverhältnissen beschränken.

4. Die Installation von Küchenmühlen ist zu untersagen bei Liegenschaften, deren Entwässerung ohne jede Reinigungsvorrichtung direkt in den Vorfluter erfolgt und dort, wo die Schüttsteinabläufe an Fettabscheider angeschlossen sind.

5. Da die Anschaffungskosten der Küchenmühlen relativ hoch sind, werden diese nur langsam und in beschränktem Umfange in Europa Eingang finden, und zwar vornehmlich in komfortableren Wohnungen. Es scheint nicht richtig zu sein, wenn die Folgen der Installation dieser Mühlen in vollem Umfang durch die Allgemeinheit getragen werden müssen. Es bleibt deshalb den Gemeindebehörden vorbehalten, für die Installation der Küchenmühlen einen einmaligen angemessenen Beitrag an die Mehrkosten der Abwasseranlagen zu verlangen, samt einer jährlichen Gebühr für evtl. vermehrte Reinigungsarbeit in den Kanälen und grössere Betriebskosten der Kläranlage. Allfällige Einnahmen aus dem Verkauf der vermehrten Schlamm- und Klärgasmenge wären billigerweise entsprechend zu berücksichtigen. Diese Gebühr wird sich in tragbarem Rahmen halten, gemessen an den Kosten der Küchenmühlen selbst.

6. Zusammengefasst können die Vor- und Nachteile, die sich aus dem Betrieb der Küchenmühlen ergeben, wie folgt einander gegenübergestellt werden:

\section{Vorteile:}

a) Hygienische.

b) Verminderung der Kehrichtabfuhr.

c) Besserer Kläreffekt in den Absetzbecken von Kläranlagen.

d) Vermehrter Gasanfall in den Faulräumen der Kläranlagen.

\section{Nacbteile:}

a) Vermehrter Wasserverbrauch.

b) Eventuell vermehrte Kanalreinigung.

c) Zusätzliche Belastung der Vorfluter bzw. bauliche Aufwendungen für Gegenmassnahmen.

d) Vermehrter Abtransport von Sand aus Kanälen und Sandfängen, vermehrte Erosion der Kanalsohlen, vermehrter Anfall an Schlamm und an organischen Stoffen im Sandfang.

e) Erforderliche Vergrösserung der projektierten bzw. vorzeitige Erweiterung bestehender Abwasserreinigungsanlagen. 
f) Verschlechterung der Gasqualität (evtl. wirtschafticher Vorteil).

g) Eventuelle Betriebsschwierigkeiten in den Faulräumen.

7. Abschliessend seien noch die Schlussfolgerungen bekanntgegeben, die von der Organisation für Europäische Wirtschaftszusammenarbeit [12] bezüglich der Kehricht-Abwasserbeseitigung für die europäischen Verhältnisse gezogen werden. Diese lauten:

a) Für die Beseitigung von Kehricht mit Abwasserschlamm können verschiedene Methoden angewendet werden. In Europa sind nur wenige Beispiele dieser Beseitigungsart bekannt, obgleich in den meisten Fällen damit bemerkenswerte Erfolge erzielt worden sind. Nur vereinzelte dieser Methoden lassen eine breitere Anwendung erwarten, mit Ausnahme vielleicht der Kompostierung.

b) Falls der Kehricht nicht zum Füttern von Schweinen benützt werden kann, bietet die Kehrichtzerkleinerung vor der Behandlung in einer zentralen Kläranlage oder vor Einbringen in die Faulräume wohl eine der zufriedenstellendsten Arten der Kehrichtbeseitigung, wobei die Küchenmühlen, abgesehen von den hohen Anschaffungskosten, wesentliche Vorteile bieten würden.

c) Sofern das Kanalnetz gut angelegt und die Kläranlage genügend gross bemessen ist, werden keine wesentlichen Schwierigkeiten durch das Einbringen zerkleinerten Kehrichts in die Abwasseranlagen zu erwarten sein.

d) Die Zahl der in Amerika installierten Küchenmühlen wird zweifellos noch zunehmen. Die allgemeine Anwendung wird durch die hohen Kosten verhindert.

e) Die Installation von Küchenmühlen in Europa wäre begrüssenswert. Es ist jedoch nur mit einer langsamen Zunahme dieser Apparate zu rechnen.

\section{IITERATURVERZEICHNIS}

[I] Haseltine, T. R., Addition of Garbage to Semage, Water Sewage Works 1950, Nr. II, 467-472.

[2] Tolman, S. L., Ground Garbage. Its Effect upon the Semer System and Semage Treatment Plant, Sewage Works J. 1947, Nr. 3, 44I-463.

[3] Owen, M. B., The Future of Home Garbage Grinders, Water Sewage Works 1949, Nr. 5, I87-I 89 .

[4] Couch, L. I., and Kulin, H. J., Municipal Garbage Disposal by Housebold Grinders at Faspar, Indiana, Sewage Ind. Wastes 22, H. 9, I I38-II I46 (I950). 
[5] Schroepfer, G. J., Minneapolis-St.-Paul-Sanitary District. Report on Effect o Addition of Ground Garbage on Semage Treatment Operation (interner Betricht vom 25. September 1944).

[6] MERKEL, W., Abschmemmung der Küchenabfälle und Zukunft des Stadtkompostes, Gesundheits-Ing., 72, I71-I72 (I95I).

[7] KuRz, H., Die Wirkung pon Regenüberfallbauwerken städtischer Kanalisationen auf die Vorfluter, Gesundheits-Ing., 46, H. 7/8, 73 und 85 (1923).

[8] BAYERLE, B., Die Verscbmutzung der Wasserläufe durch die Regenauslässe der lintmässerungsnetze, Gesundheits-Ing., 61, H. 30, 4I3-417 (1938).

[9] Hörler, A., DieWirkung der Regenauslässe, Schweiz. Bauzeitung, 11 8, Nr. 20 (I94I).

[Io] BREnTano, M., Untersucbungen über die Abwasserverbältnisse von Zürich, Diss. (ETH 1934).

[I I] Müller-Neuhaus, G., Zur Frage der Bemessung pon Regenüberfällen bei Entwässerungsnetzen und Kläranlagen, Gesundheits-Ing., 71, H. 9/10, I49-152 (1950).

[12] Adduction d'Eau. Traitement des Effluents Urbains, Organisation Européenne de Coopération Economique, I953, I29-I33. 